\title{
The correlation between solvent treatment and the microstructure of PAN-b-PEG copolymer membranes
}

\author{
Fuwei Lu, Ying Kong, Hongling Lv, Jingrong Yang and Zhaoxuan Feng
}

Block copolymer membranes consisting of thiophene-permselective components for use in gasoline desulfurization were prepared by the copolymerization of polyethylene glycol (PEG) with acrylonitrile by water-phase precipitation copolymerization. In this study, a solvent annealing treatment was applied to evaluate the microdomain orientation and the affinity between polyacrylonitrile-block-polyethylene glycol (PAN-b-PEG) copolymers and solvents. Using atomic force microscopy, appreciable microphase separation was observed in the PAN-b-PEG membranes. The affinity of the PEG segments and solvents was reflected by the different sizes of the PEG domains. The sizes of the PEG domains grew gradually with solvent annealing treatments of heptane, toluene and thiophene for all of the copolymers. Using phase separation by different solvent annealing treatments, PAN-b-PEG could be used as a highly selective material for thiophene. This method can also be applied as an important alternative to provide a semi-quantitative method for determining the adsorption properties of pervaporation membranes, which cannot be evaluated by sorption experiments.

Polymer Journal (2011) 43, 378-384; doi:10.1038/pj.2011.6; published online 16 February 2011

Keywords: gasoline desulfurization; microphase separation; pervaporation; polyacrylonitrile-block-polyethylene glycol (PAN-b-PEG)

\section{INTRODUCTION}

Pervaporation (PV) is a liquid separation process in which liquid is in contact with the membrane at the feed side, whereas the permeate side is kept dry and at low pressure. ${ }^{1}$ The discriminating affinity that exists between the feed components and the polymeric membrane results in the partitioning of mixtures and separation. ${ }^{2}$ Many liquid organicorganic mixtures have been successfully separated with the PV process, such as in the process of gasoline desulfurization. ${ }^{3-6}$ In gasoline desulfurization by PV, a non-porous polymeric membrane usually serves as the separating barrier for the process. ${ }^{7}$ When the membrane is in contact with the gasoline feed, sulfur components can be preferentially removed from the feed because of their higher affinity with and/or their quicker diffusivity through the membrane.

In this technique, the polymer membrane is the central focus of the research and development. The selection of the polymer material is critical to the success of the PV separation. ${ }^{8}$ Because gasoline desulfurization is an emerging application of PV, the guidelines for membrane material selection are crude, and predictive methods are still under development. In a previous study of membrane material selection, much attention was given to the relationships describing the mass transfer properties of polymers. ${ }^{9}$ The common approaches focus on the use of empirical parameters, and the most widely used parameters remain the well-known solubility parameters. ${ }^{10,11}$ Lin et al. ${ }^{12-15}$ investigated the PV characteristics of polyethylene glycol
(PEG) membranes on real gasoline desulfurization using the solubility parameters theory. Qi et al. ${ }^{16}$ evaluated the desulfurization performance of polydimethylsiloxane membranes. Each studied membrane showed sound separation performance; however, reports on the sorption and transport behavior based on the solubility parameters of the membrane material selection are insufficient. The sorptiondiffusion properties of membranes should be tested experimentally before being installed in a PV unit.

Until recently, the sorption-diffusion information of the individual penetrants has always been provided by sorption experiments. ${ }^{17}$ Our previous work has reported the solubility and diffusion behavior of typical gasoline components through PEG membranes by sorption experiments. ${ }^{18}$ The detailed study of the solution of gasoline molecules on the PEG membrane has provided information that is helpful in optimizing membrane performance, but sorption experiments performed with immersion tests could not meet the accuracy of the variation of the weight change, especially for polymers with good solvent-resistance properties. For example, the fractional weight change of the polyacrylonitrile-block-polyethylene glycol (PAN-bPEG) copolymer after being immersed in solvents is hardly measurable because of the small weight change that occurs after swelling in gasoline components. In addition to the sorption experiments, the solubility of PEG chains and gasoline components can also be investigated by the morphology of PEG aggregations. When the 
interaction between the membrane and the adsorbing solvent is important for separation, the nature of the solvent reveals information about the membrane. Penetrants are widely used as probes to infer the detailed structure of polymer systems. ${ }^{19,20}$ However, there is no direct method to evaluate the affinity between solvents and polymers.

This paper attempts to correlate the selectivity of different gasoline components with the morphology variation in the microphase separation of PAN-b-PEG block copolymers. The correlation between gasoline components and PAN-b-PEG block copolymers is determined by solvent annealing experiments.

\section{EXPERIMENTAL PROCEDURE}

Materials

Two PEG samples with molecular weights of 2000 and $20000 \mathrm{Da}$ were purchased from Shanghai Ace of reagents (Shanghai, PR China). Analysisgrade acrylonitrile was purchased from Kermel Chemical Reagent (Tianjin, PR China). Ceric ammonium nitrate was purchased from Shanghai Chemical Reagent (Shanghai, PR China). Thiophene was purchased from Alfa Aesar (New York, NY, USA). N,N-Dimethylformamide, toluene and heptane were purchased from Sinpharm Chemical Regent (Hangzhou, PR China). All of the solvents were used without further purification.

\section{Polymerization}

A series of PEG-b-PAN copolymers with various molecular weights of PEG were synthesized by water-phase precipitation copolymerization using ceric (IV) ammonium nitrate as an initiator. The methodology of copolymerization can be found elsewhere. ${ }^{21,22}$

\section{Film formation}

The polymer was dissolved in N,N-Dimethylformamide to obtain a $1 \mathrm{wt} \%$ solution, which was then filtered through a porous polytetrafluoroethylene membrane (with $0.25-\mu \mathrm{m}$ diameter pores). The films were prepared by spin coating at 2000 r.p.m. for $30 \mathrm{~s}$ on freshly cleaved mica plates after heating the solution to its boiling point. After spinning, the films were first air-dried for $24 \mathrm{~h}$ and then dried under vacuum at room temperature for another $12 \mathrm{~h}$. The spun membranes were applied in solvent annealing experiments.

\section{Characterization}

The powdery copolymers were ground in an agate mortar, and their elemental composition was detected by X-ray photoelectron spectroscopy. The X-ray photoelectron spectroscopy was performed using a PHI5000C ESCA System with a Magnesium KR X-ray source $(1253.6 \mathrm{eV})$ (PerkinElmer Inc., Waltham, MA, USA). The X-ray gun was operated at a power of $250 \mathrm{~W}$, and the maximum voltage was maintained at $140 \mathrm{kV}$ with a detection angle of $54^{\circ}$. The chamber pressure during analysis was $\sim 1 \times 10^{-8}$ Torr. Fourier transform infrared (FT-IR) spectra of the copolymers were obtained on a Nicolet Avatar 370 FT-IR spectrometer (Thermo Fisher Scientific Inc., Waltham, MA, USA).

\section{Solvent annealing experiment}

The spun films were placed in an airtight vessel with $0.1 \mathrm{ml}$ of singlecomponent gasoline at the initiation of the solvent annealing process. The temperature of the model gasoline component's vapor was kept at $333.15 \mathrm{~K}$ for $2 \mathrm{~h}$. After being exposed to model gasoline component vapor, the samples were quickly removed from the vessels and dried under vacuum at room temperature. The samples were investigated with atomic force microscopy (AFM).

\section{Sorption experiment}

During the sorption procedure, single-component liquid sorption was measured by immersing membrane pieces of PEG in a solvent. The change in membrane weight was determined. ${ }^{18}$ The constant, K, which depends on the structural characteristics of the polymer and provides information about the interaction between polymer and solvent, can be found using an empirical formula. ${ }^{23}$

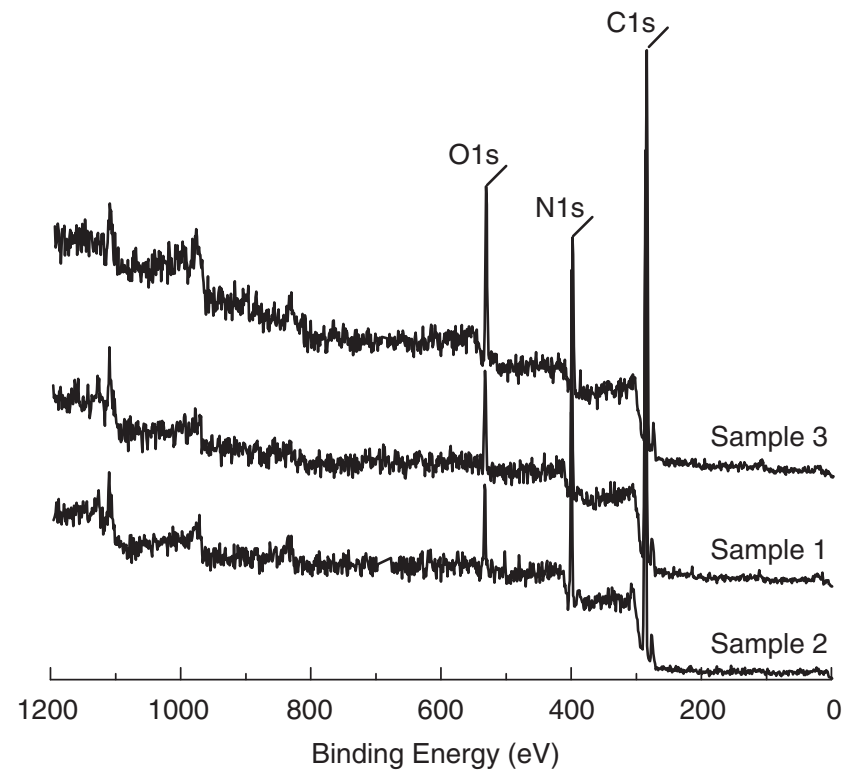

Figure 1 X-ray photoelectron spectroscopy core level spectra of polyacrylonitrileblock-polyethylene glycol copolymers.

Table 1 A summary of element weight percentages and PEG contents in PAN-b-PEG copolymers

\begin{tabular}{|c|c|c|c|c|c|}
\hline \multirow{2}{*}{$\begin{array}{l}\text { Sample } \\
\text { number }\end{array}$} & \multirow{2}{*}{$\begin{array}{c}\text { Molecular weight } \\
\text { of PEG }\end{array}$} & \multicolumn{3}{|c|}{$\begin{array}{l}\text { Weight percentage } \\
\text { of element (wt\%) }\end{array}$} & \multirow{2}{*}{$\begin{array}{c}\text { PEG in copolymer } \\
\text { (wt\%) }\end{array}$} \\
\hline & & $C$ & 0 & $N$ & \\
\hline 1 & 2000 & 74.59 & 6.32 & 15.07 & 17.93 \\
\hline 2 & 20000 & 78.71 & 5.46 & 15.83 & 15.02 \\
\hline 3 & 2000 & 73.80 & 11.17 & 12.36 & 31.57 \\
\hline
\end{tabular}

Abbreviations: PAN-b-PEG, polyacrylonitrile-block-polyethylene glycol; PEG, polyethylene glycol.

\section{RESULTS AND DISCUSSION}

\section{Characterization of the PAN-b-PEG copolymers}

Samples 1 and 3 are copolymers prepared with different PEG content of PEG 2000. Sample 2 is the copolymer formed by the copolymerization of PEG 20000 and acrylonitrile. Figure 1 shows the X-ray photoelectron spectroscopy spectra of the elemental composition of the PAN-b-PEG copolymers. In the case of the three copolymers, three strong peaks at 285, 401 and $525 \mathrm{eV}$ were observed, which were attributed to C1s, N1s and O1s, respectively. There were no foreign element peaks found for any of the copolymers. The elemental compositions of carbon $(\mathrm{C})$, oxygen $(\mathrm{O})$ and nitrogen $(\mathrm{N})$ of all the samples were calculated and are presented in Table 1. Because PEG is the only source of oxygen for the synthesized copolymers, the measurement of elemental composition provides valuable information for calculating the weight percentages of PEG in PAN-b-PEG copolymers. The PEG contents in samples 1 and 3 were 17.93 and $31.57 \mathrm{wt} \%$, respectively. The PEG content in sample 2 was $15.02 \mathrm{wt} \%$, similar to the content of sample 1 .

Figure 2 shows the FT-IR spectra of the studied copolymer membranes. It can be seen that, as compared with the FT-IR spectrum of PEG 2000, there is a series of new absorption peaks at 2246.89, 2937.56 and $1452.00 \mathrm{~cm}^{-1}$ in the spectra of the copolymer membranes. These peaks were assigned to $v(\mathrm{CN}), v_{\mathrm{as}}\left(\mathrm{CH}_{2}\right)$ and $\sigma\left(\mathrm{CH}_{2}\right)$ on 
the polyacrylonitrile segment in the PAN-b-PEG block copolymers, respectively. The difference of PEG content in the PAN-b-PEG block copolymers can be fully embodied by the adsorption peak of $v(\mathrm{C}-\mathrm{O}-\mathrm{C})$ at $1109.78 \mathrm{~cm}^{-1}$. The content of the $\mathrm{C}-\mathrm{O}-\mathrm{C}$ group increases gradually from sample 2 to 1 to 3 , which is consistent

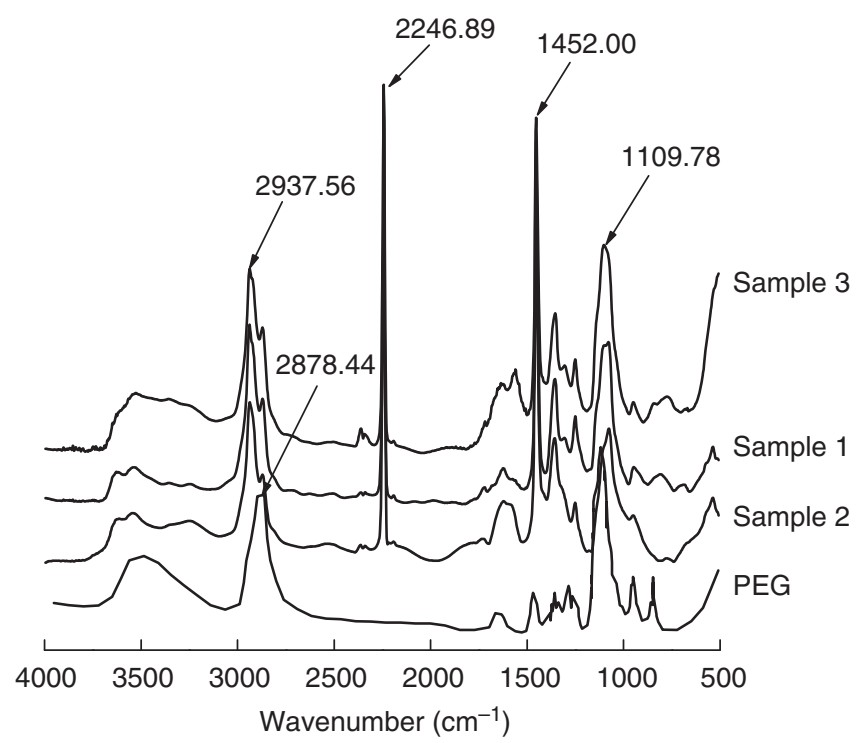

Figure 2 Fourier transform infrared spectra of polyacrylonitrile-blockpolyethylene glycol (PAN-b-PEG) copolymers.
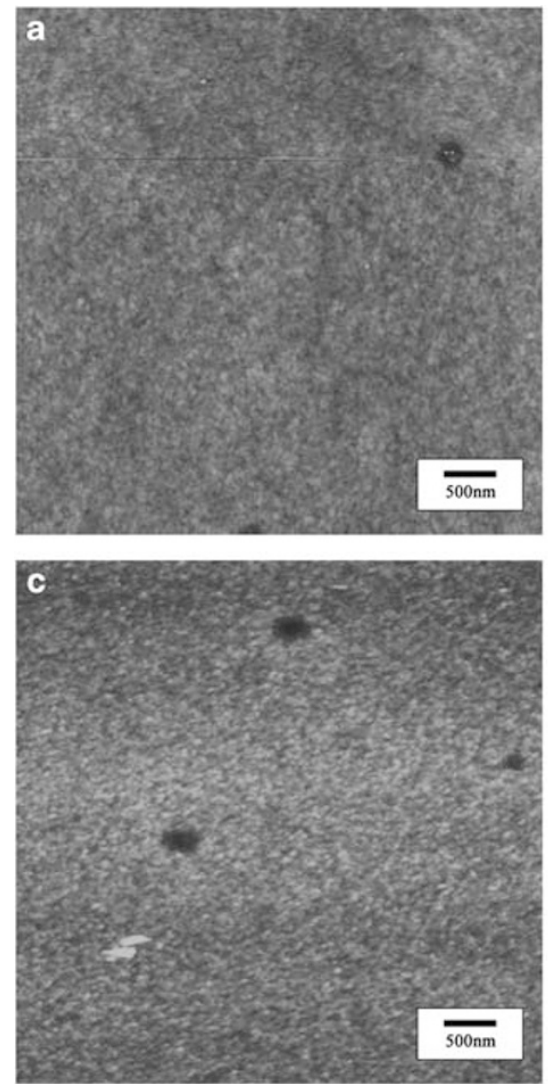

with the elemental composition analysis of the X-ray photoelectron spectroscopy spectra.

\section{Film morphologies annealed by solvents}

For a given system, a solvent that is appropriate for one block can be classified as neutral, slightly selective or strongly selective, according to whether it is appropriate for one block in copolymer, or a nonsolvent for the other block. Thiophene, toluene and heptane serve as the annealing solvents. Thiophene and toluene are good solvents for the PEG segment in a PAN-b-PEG block copolymer. The PAN segments are insoluble in all of the solvents. For a specific solvent, there are visible differences in polymer-solvent affinity between the different polymer segments. These differences might be related to the difference in the affinity of the solvent to a specific block, especially to the PEG segments.

The phase images of thin-films of the copolymer of sample 1 with $17.93 \mathrm{wt} \%$ PEG are shown in Figure 3. The microphase separation morphologies of the annealed samples using gasoline components under the same annealing conditions were investigated by AFM. The micrographs demonstrate that the PAN-b-PEG membranes have distinct phases of microphase separation, consisting of a PEG phase and a PAN phase. Different groups have already demonstrated the concentration of one block inside the holes triggered by the interaction of the special segment with the solvent vapor. The light region is the bulk PAN phase, and the dark region is the aggregation of the dispersed PEG segments, displayed as holes in the AFM images. In particular, there is no obvious phase separation by thermal treatment in Figure 3a. On exposure to heptane vapor, the PEG chains aggregate into the dark phase in Figure $3 \mathrm{~b}$. The dispersed PEG
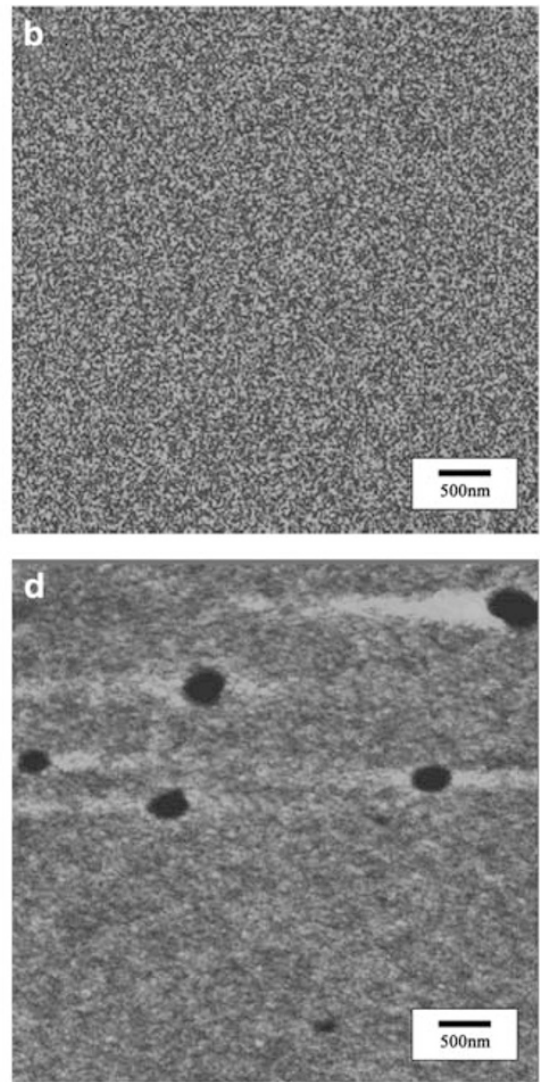

Figure 3 Phase images of sample 1 annealed by gasoline components: (a) blank; (b) heptane; (c) toluene; and (d) thiophene. 

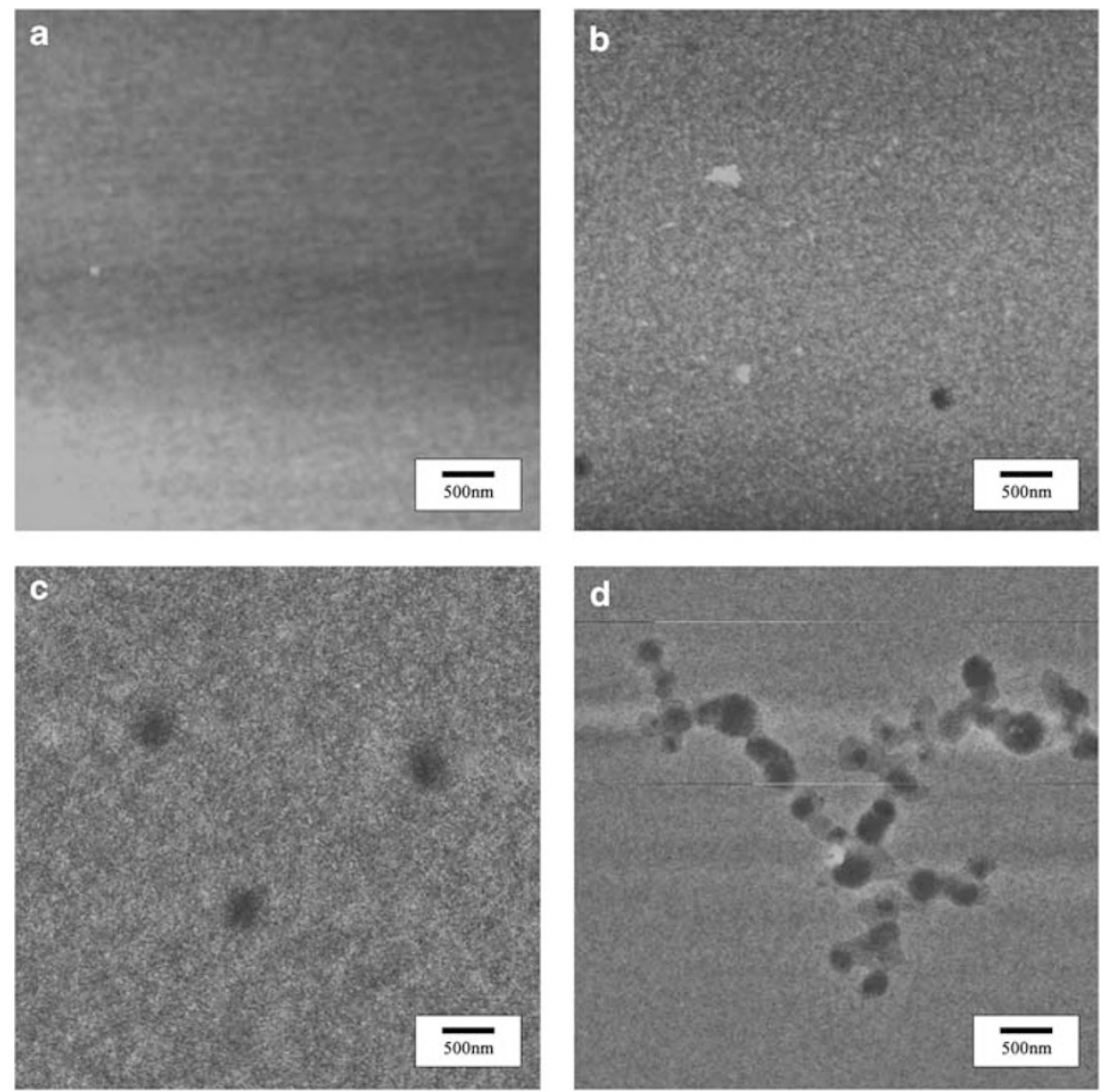

Figure 4 Phase images of sample 2 annealed by gasoline components: (a) blank; (b) heptane; (c) toluene; and (d) thiophene.

domains aggregated and made larger domains when the copolymer membranes were annealed by toluene vapor. For thiophene vapor annealing treatment, some of the PEG chains aggregated into pores with an average diameter of about $350 \mathrm{~nm}$, a larger pore size than samples annealed by heptane or toluene vapor. The size of the PEG pores aggregated by solvent annealing may be related to the affinity between the polymer and solvent. The image processing demonstrates that the sizes of PEG domains grow gradually with solvent annealing treatments of heptane, toluene and thiophene.

\section{The influence of PEG molecular weight}

Figure 4 is an AFM image of the surface topography of the annealed membranes of sample 2. Figure 4 a shows that the surface of the film is smooth and featureless because of thermal annealing, indicating that no microphase separation exists to form PEG pores at the film surface after the formation of the layer. Rare pores of PEG domains on a smooth background can be observed in Figure $4 \mathrm{~b}$, which was annealed by heptane vapor. The larger sizes of the pores aggregated by toluene vapor annealing and the selective swelling effect of the solvent vapor lead to a rougher film morphology as compared with the films exposed to heptane. In the presence of thiophene, the swollen PEG chains become more mobile and can more easily reorganize themselves, and the PEG domains migrate together to form larger aggregations, as shown in Figure 4d. The image processing demonstrates that the sizes of the PEG domains grow gradually with the solvent annealing treatments of heptane, toluene and thiophene, consistent with the results of the images of sample 1. Generally, the boundary between the PEG aggregates and the bulk PAN regions becomes unclear as the lengths of PEG chains increase from 2000 to
20000 Da with similar PEG content. This ambiguity might be due to the fact that the PEG chains with a molecular weight of $20000 \mathrm{Da}$ are slower than the PEG $2000 \mathrm{Da}$ chains under the same solvent annealing conditions; however, the reason for the aggregation of the PEG clusters with thiophene vapor annealing treatment requires further investigation.

\section{The influence of PEG weight content}

Figure 5 displays the phase images of thin-films of the copolymer of sample 3 with $31.57 \mathrm{wt} \%$ PEG 2000. It can be seen that PEG segments are highly dispersed in bulk PAN. When the film was treated by thermal treatment or solvent annealing, microphase aggregation occurred. No obvious differences were observed between heptane vapor annealing and thermal treatment without a solvent. The poor affinity of heptane to both the PEG and PAN block should be noted, which was consistent with the results of the images of samples 1 and 2 . The dispersed PEG domains aggregate and form larger domains under toluene vapor annealing treatment. The hybrid structure of the microphase separation morphology of the PEG domains with different sizes might be a consequence of the affinity of the PEG domains with toluene or the time limitation of $2 \mathrm{~h}$. For thiophene vapor annealing treatment, clear borders between the PEG and PAN block were apparent in the images of films, which was different from the morphology of samples 1 and 2. A smooth surface on the bulk PAN phase can be observed because of the high aggregation of PEG blocks by thiophene vapor annealing. On thiophene vapor exposure, the crystallization of PEG segments can be obtained from the peaks by the high aggregation of the PEG blocks. The surface morphology changed from holes to island-like structures (with heights of around $4 \mathrm{~nm}$ ), 

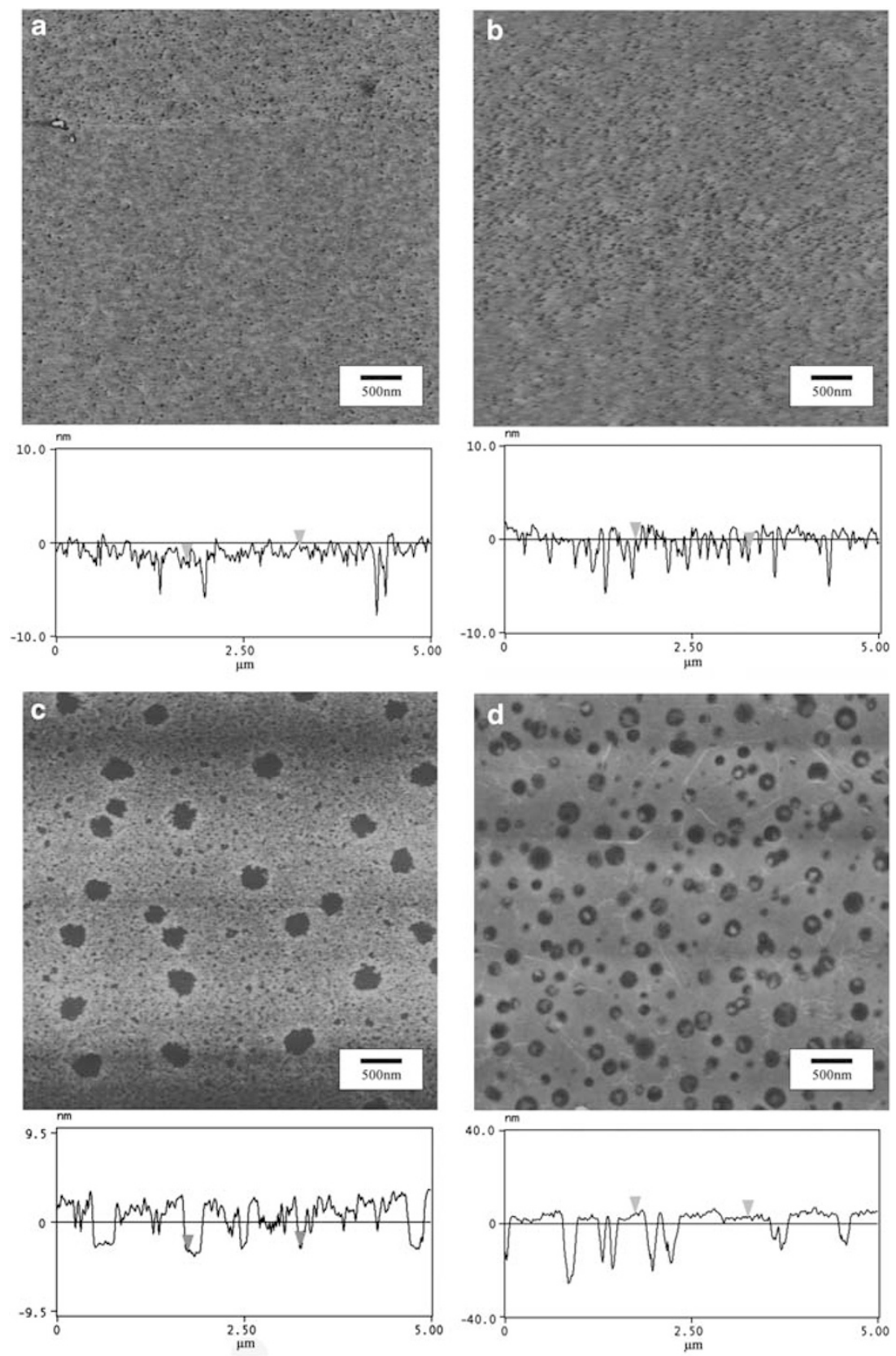

Figure 5 Phase images of sample 3 annealed by gasoline components: (a) blank; (b) heptane; (c) toluene; and (d) thiophene.

with an average number of islands or peaks similar to the number of holes. The formation of islands as replacements for holes indicates that the PEG block quantity increases substantially on the pore surface on thiophene vapor annealing, because thiophene may significantly swell the PEG blocks; however, the crystallization was not observed in the samples subjected to heptane and toluene vapor annealing treatment.

Pioneering work in exploiting solvent annealing by different gasoline components has demonstrated that various film morphologies can be obtained by varying the annealing solvents. On the basis of the effective interactions between the PEG and the other domains, a possible mechanism of the morphologies formation has been suggested. When the films are annealed under a saturated solvent vapor environment, the top surface is cooled because of the evaporation of the solvent. The enhanced polymer mobility caused by the solvent swelling can lead to a high degree of ordering in the film. ${ }^{24}$ However, the ordering degree of the morphologies should be primarily determined by the species of the solvents and other annealing treatment conditions. Toluene and thiophene are selective solvents for PEG blocks. Under the saturated solvent vapor, the disordered phase of the PEG blocks is highly swollen and is driven into a high degree of ordering in the film. This scenario of the PEG aggressions annealed by different gasoline components is illustrated schematically in Figure 6. As the solvent evaporates, an ordering front will propagate into the film, leading to a perpendicular orientation of PEG cylindrical microdomains throughout the film. The crystal growth restriction of the PEG blocks is a consequence of the formation of a glassy PAN layer adjacent to the PEG chains. In this case, thiophene has prior affinity to 


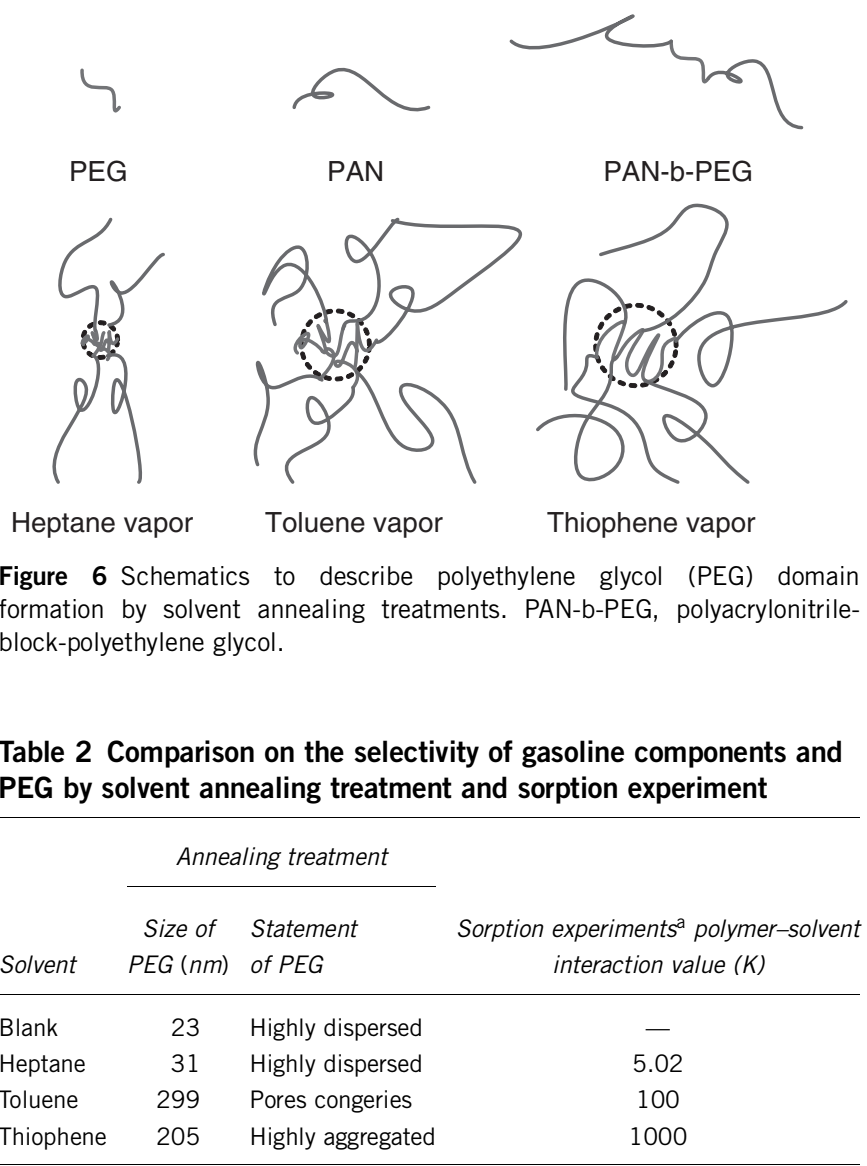

Abbreviation: PEG, polyethylene glycol.

aThe value of $\mathrm{K}$ was obtained from reference 18

PEG blocks and can reduce the restriction of PEG crystalline structure formation.

\section{Comparison with sorption experiment results}

From the above surface topography discussions, thiophene was the preferential sorption component in PEG blocks. To correlate the selectivity of gasoline components and the morphology variation of the PEG blocks, the difference in the constant polymer-solvent interaction value $(\mathrm{K})$ calculated by sorption experiments ${ }^{18}$ and the size variation of the PEG pores of sample 3, treated under the same temperature, are displayed in Table 2. The size variation of the PEG pores demonstrates that the morphology of the microphase separation is strongly dependent on the species of the gasoline components, which show the same polymer-solvent interaction tendency of PEG by the sorption experiments. The coalescence circumstance of multiple small aggregations of the PEG domains with toluene vapor and the affinity between toluene and PEG blocks affect the coalescence of the PEG domains. The average diameter of the coalescence domains is $\sim 299 \mathrm{~nm}$, more than 10-fold larger than the domains created by heptane vapor annealing treatment. A variation of the $\mathrm{K}$ value similar to that obtained by the sorption experiments could also be observed; however, the arrangement of the PEG domains annealed by thiophene vapor was more effective than that of other gasoline components, showing no small PEG pores dispersed in the bulk PAN phase. The lack of pores might be caused by the strong affinity of thiophene to the PEG domains. The high value of $\mathrm{K}$ in the thiophene sorption experiments showed the normal swelling properties of PEG mem- branes. Thus, the solvent annealing treatment could be used as a semiquantitative method for studying the adsorption properties of polymeric membranes, which could not be analyzed by sorption experiments. As discussed above, PAN-b-PEG membranes were fabricated for a desulfurization process by PV. Compared with the PAN-b-PEG membranes, no permeate sample could be collected by the PAN membrane without PEG blocks, which means that PEG microdomains function as the permeate sample carrier. The selectivity factor of all the samples was higher than 2.0 for thiophene in the gasoline component mixtures, ${ }^{12}$ which resulted in PEG being the selective segment for gasoline desulfurization in the copolymer.

\section{CONCLUSION}

In this work, the microphase separation of PAN-b-PEG copolymers was investigated by solvent annealing treatment. The size of the PEG domains grows gradually with the solvent annealing treatments of heptane, toluene and thiophene for all of the copolymers. Thiophene shows previous affinity to PEG blocks of PAN-b-PEG copolymers. On the basis of the size variation of the PEG domains, this method can be used as a semi-quantitative method to determine the adsorption properties of the copolymer membranes. The PEG domains are key components for gasoline desulfurization by PV processes. The morphology of PEG aggregations annealed by the mixture of gasoline components might be useful in further understanding the sulfur removing process. The correlation between desulfurization performance and membrane morphology should also be considered in future studies.

\section{ACKNOWLEDGEMENTS}

The authors gratefully acknowledge the support from the Program for New Century Excellent Talents in the University of the Ministry of Education of China (NCET-06-0605), the Innovation Team 'Green Chemistry and Engineering of Efficient Transformation of Heavy Oil' of the Ministry of Education of China, the Research Fund for the Doctoral Program of Higher Education (20090133120008) of the Ministry of Education of China, the Natural Science Foundation of the Shandong Province of China (ZR2009BQ011) and the Open Fund from the State Key Laboratory of Hollow Fiber Membrane Materials and Processes (201042).

1 Smitha, B., Suhanya, D., Sridhar, S. \& Ramakrishna, M. Separation of organic-organic mixtures by pervaporation-a review. J. Membr. Sci. 241, 1-21 (2004).

2 Jiang, L., Wang, Y., Chung, T., Qiao, X. \& Lai, J. Polyimides membranes for pervaporation and biofuels separation. Prog. Polym. Sci. 34, 1135-1160 (2009).

3 Yildirim, A., Hilmioglu, N. \& Tulbentci, S. Separation of benzene/cyclohexane mixtures by pervaporation using PEBA membranes. Desalination 219, 14-25 (2008).

4 Ray, S. \& Ray, S. K. Synthesis of highly methanol selective membranes for separation of methyl tertiary butyl ether (MTBE)-methanol mixtures by pervaporation. J. Membr. Sci. 278, 279-289 (2006).

5 Yang, L., Kang, Y., Wang, Y., Xu, L., Kita, H. \& Okamoto, K. Synthesis of crown ethercontaining copolyimides and their pervaporation properties to benzene/cyclohexane mixtures. J. Membr. Sci. 249, 33-39 (2005).

6 Semenova, S. Polymer membranes for hydrocarbon separation and removal. J. Membr. Sci. 231, 189-207 (2004).

7 Shao, P. \& Huang, R. Polymeric membrane pervaporation. J. Membr. Sci. 287, 162-179 (2007).

8 Mauviel, G., Berthiaud, J., Vallieres, C., Roizard, D. \& Favre, E. Dense membrane permeation: from the limitations of the permeability concept back to the solutiondiffusion model. J. Membr. Sci. 266, 62-67 (2005).

9 Shah, M., Noble, R. \& Clough, D. Analysis of transient permeation as a technique for determination of sorption and diffusion in supported membranes. J. Membr. Sci. 280, 452-460 (2006)

10 Liu, Y. \& Shi, B. Hollow fiber supported liquid membrane for extraction of ethylbenzene and nitrobenzene from aqueous solution: a Hansen solubility parameter approach. Sep. Purif. Technol. 65, 233-242 (2009).

11 Mandal, S. \& Pangarkar, V. Development of co-polymer membranes for pervaporative separation of methanol from methanol-benzene mixture-a solubility parameter approach. Sep. Purif. Technol. 30, 147-168 (2003). 
12 Lin, L., Kong, Y., Wang, G., Qu, H., Yang, J. \& Shi, D. Selection and crosslinking modification of membrane material for FCC gasoline desulfurization. J. Membr. Sci. 285, 144-151 (2006).

13 Lin, L., Zhang, Y. \& Kong, Y. Pervaporation separation of n-heptane/thiophene mixtures by polyethylene glycol membranes: modeling and experimental. J. Colloid Interface Sci. 339, 152-159 (2009).

14 Lin, L., Kong, Y., Xie, K., Lu, F., Liu, R., Guo, L., Shao, S., Yang, J., Shi, D. \& Zhang, Y. Polyethylene glycol/polyurethane blend membranes for gasoline desulphurization by pervaporation technique. Sep. Purif. Technol. 61, 293-300 (2008).

15 Lin, L., Wang, G., Qu, H., Yang, J., Wang, Y., Shi, D. \& Kong, Y. Pervaporation performance of crosslinked polyethylene glycol membranes for deep desulfurization of FCC gasoline. J. Membr. Sci. 280, 651-658 (2006).

16 Qi, R., Wang, Y., Li, J., Zhao, C. \& Zhu, S. Pervaporation separation of alkane/thiophene mixtures with PDMS membrane. J. Membr. Sci. 280, 545-552 (2006).

17 Shah, M., Noble, R. \& Clough, D. Measurement of sorption and diffusion in nonporous membranes by transient permeation experiments. J. Membr. Sci. 287, 111-118 (2007).

18 Lin, L., Kong, Y. \& Zhang, Y. Sorption and transport behavior of gasoline components in polyethylene glycol membranes. J. Membr. Sci. 325, 438-445 (2008).
19 Krea, M., Roizard, D., Moulai-Mostefa, N. \& Sacco, D. New copolyimide membranes with high siloxane content designed to remove polar organics from water by pervaporation. J. Membr. Sci. 241, 55-64 (2004).

20 Guo, R., Huang, H., Du, B. \& He, T. Solvent-induced morphology of the binary mixture of diblock copolymer in thin film: the block length and composition dependence of morphology. J. Phys. Chem. B. 113, 2712-2724 (2009).

21 Su, Y., Cheng, W., Li, C. \& Jiang, Z. Preparation of antifouling ultrafiltration membranes with poly (ethylene glycol)-graft-polyacrylonitrile copolymers. J. Membr. Sci. 329, 246-252 (2009).

22 Fernandez, M. D. \& Guzman, G. M. Aqueous polymerization of methyl Methacrylate initiated by Ce (IV)-alcohol redox systems: effect of acid concentration and additives. J. Polym. Sci. Part A Polym. Chem. 27, 2427-2433 (1989).

23 Kumar, H. \& Siddaramaiah A study of sorption/desorption and diffusion of substituted aromatic probe molecules into semi interpenetrating polymer network of polyurethane/ polymethyl methacrylate. Polymer 46, 7140-7155 (2005).

24 Bang, J., Jeong, U., Ryu, D. Y., Russell, T. \& Hawker, C. J. Block copolymer nanolithography: translation of molecular level control to nanoscale patterns. Adv. Mater. 21, 1-24 (2009). 